Boletín de la Sociedad Botánica de México 52: 121-124, 1992

DOI: $10.17129 /$ botsci.1409

Bol. Soc. Bot. México 52:121-124 (1992)

\title{
Fisiones céntricas en cromosomas metacéntricos de Nothoscordum bivalve (Alliaceae) de México
}

El género Nothoscordum Kunth pertenece a la familia Alliaceae (Dahlgren y Clifford, 1982). Existen numerosos estudios citogenéticos en Nothoscordum fragrans Kunth y en el complejo poliploide de $N$. inodorum (Soland. ex Aiton) Nich. Estos estudios realizados en poblaciones silvestres de Estados Unidos de Norteamérica, Uruguay y en clones cultivados en Japón, mostraron variaciones en el número y en la forma de los cromosomas somáticos $(2 n)$ y de los cromosomas de los granos de polen (n) entre y dentro de las especies del complejo N. inodorum (Núñez, et al., 1974), así como en plantas de N. fragrans y sus clones (Levan y Emsweller, 1938; Garber, 1944; Sato y Asano, 1951; Núñez, et al., 1972; Nassar y deAguiar, 1978).

Nothoscordum bivalve (L.) Britt., tiene $2 n=18$ y un cariotipo formado por $14 \mathrm{M}+4 \mathrm{~T}$ (Jones, 1978) y no se ha referido hasta el momento un fenómeno similar al encontrado en otras especies. En el origen de esta especie, se considera que han intervenido tanto la hibridación como la poliploidía (Núñez, et al., 1974 y Jones, 1978) y la fusión-fisión de cromosomas (fusiones y fisiones robertsonianas).

El trabajo estuvo enfocado al análisis de los cromosomas somáticos $(2 n)$ de los bulbos y de los cromosomas mitóticos (n) de los granos de polen en una población mexicana de Nothoscordum bivalve (L) Brit., así como de su progenie, generada vegetativamente de bulbillos.

Las plantas fueron colectadas a $20 \mathrm{Km}$ al oeste de Temascaltepec, Estado de México; depositandose los ejemplares de respaldo en el Herbario Nacional (MEXU), G. Palomino 056, A. Kenton y P. Rudall. Se provee evidencia de la presencia de fisiones céntricas espontáneas en plantas originales y clonadas de esta especie.

Se analizaron siete plantas originales y al menos un clon en cinco de éstas y tres o cuatro, en dos de ellas. Los clones se generaron espontáneamente en los invernaderos del Jardín Botánico del Instituto de Biología, UNAM. Se analizaron entre 10 y 20 células somáticas por cada planta original y de 14 a 24 por cada clon; así como 10 granos de polen de una planta original y de cada uno de los tres clones que florecieron en los invernaderos.

El número diploide $(2 n)$ se obtuvo en células de meristemos radiculares y el número haploide $(n)$, en la primera mitosis de los granos de polen. Los cromosomas somáticos se tiñeron en una solución de Schiff, elaborada a base de fuccina básica según García (1990), y los cromosomas de los granos de polen fueron coloreados en fresco con aceto-orceína. 
La descripción de los cariotipos está basada en Naranjo, et al. (1986). Las plantas originales mostraron $2 n=18$ y un cariotipo formado de $14 \mathrm{M}+4 \mathrm{~T}$ en el $85 \%$ de las células meristemáticas (fig. 1A y 1D). El número haploide observado fue de $n=9(7 \mathrm{M}+2 \mathrm{~T})$, tanto en las plantas originales como en los clones (fig. 1B). El 15\% de las células de las plantas originales también presentaron $2 n=19$, constituido por $13 \mathrm{M}+6 \mathrm{~T}$. El número total de brazos mayores del complemento fue, de n.f. $=32$, para células con $2 n=18$ y $2 n=19$.

El $47 \%$ de las células somáticas de los clones presentaron $2 n=18$ y un cariotipo conformado por $14 \mathrm{M}+4 \mathrm{~T}(\mathrm{n} . \mathrm{f} .=32)$. El $53 \%$ restante presentó fisiones céntricas y números cromosómicos variables. El mayor porcentaje (18\%), correspondió a células con $2 n=19$ (fig. 1C) formado de $13 \mathrm{M}+6 \mathrm{~T}$ y manteniéndose el n.f. $=32$.

En porcentajes del cinco a siete se encontraron células con $2 n=20,21,22$ y 24 . Del total muestreado, tres células presentaron $2 n=32,34$ y 48 .

Los resultados obtenidos muestran que los cromosomas de $N$. bivalve tienen un comportamiento similar al encontrado para $N$. fragrans y el complejo $N$. inodorum en los bulbos y bulbillos, no así en los granos de polen.

Esta conducta cromosómica no había sido observada en esta especie. Esto es indicativo de que las fisiones espontaneas de cromosomas metacéntricos a nivel del centrómero son un fenómeno común en el género, como ya había sido señalado por otros autores (Nassar y deAguiar, 1978). Asimismo, resulta aparente que dichas fisiones son mas comunes en clones que en plantas originales.

AGRADECIMIENTOS. Este trabajo fue realizado parcialmente por el apoyo financiero del Consejo Nacional de Ciencia y Tecnología (CONACyT), donativo clave PCCNCNA-022645. Agradecemos a Javier Martínez Ramón y a Pedro Mercado la obtención de las fotografías de este trabajo.

\section{LITERATURA CITADA}

DAHLGREN, R.M.T.y H. T. CLIFFORD. 1982. The monocotyledons: a comparative study. Academic Press, New York. 378p.

GARBER, E. 1944. Spontaneous alterations of chromosome morphology in Nothoscordum fragrans. Amer. J. Bot. 31:161-165.

GARCÍA, V.A. 1990. Técnicas y procedimientos de citogenética vegetal. Colegio de Postgraduados, Chapingo, México. Tercera edición. 144p.

JONES, K. 1978. Aspects of chromosome evolution in higher plants. En: Woolhouse, H.W. (Ed.) Advances in botanical research. v. 6 Academic Press, London. pp.177-179.

LEVAN, A. y S.L. EMSWELLER. 1938. Structural hybridity in Nothoscordum fragrans and the origin of terminally attached chromosomes. J. Heredity 29:283-299.

NARANJO, C.A., L. PogGiO y P.E. Brandham. 1986. A new template for direct morphological classification of chromosomes. Darwiniana 27(1-4):39-41.

NASSAR, N.M.A. y M.L.R. DE AGUIAR. 1978. Instability of chromosome number of Nothoscordum fragrans Kunt. Cytologia 43:371-376.

NúNEEZ, O., N. FRAYSSINET y R.H. RODRÍGUEZ. 1972. Los cromosomas de Nothoscordum Kunth (LILIACEAE). Darwiniana 17:243-245. 
NúÑEZ, O., N. FrAYSSINET, R.H.RODRÍGUEZy K.JONES. 1974. Cytogenetic studies in the genus Nothoscordum Kunth I. The N. inodorum polyploid complex. Cariologia 27(4):403-441.

SATO, D. y R. ASANO. 1951. Basikaryotype analysis in Nothoscordum fragrans. Bot. Mag. Tokyo 64:761-762.

Guadalupe Palomino, Virginia Romo, Laboratorio de Citogenética. Jardín Botánico, Instituto de Biología y ROCIO RUENES, Facultad de Ciencias, Universidad Nacional Autónoma de México. 

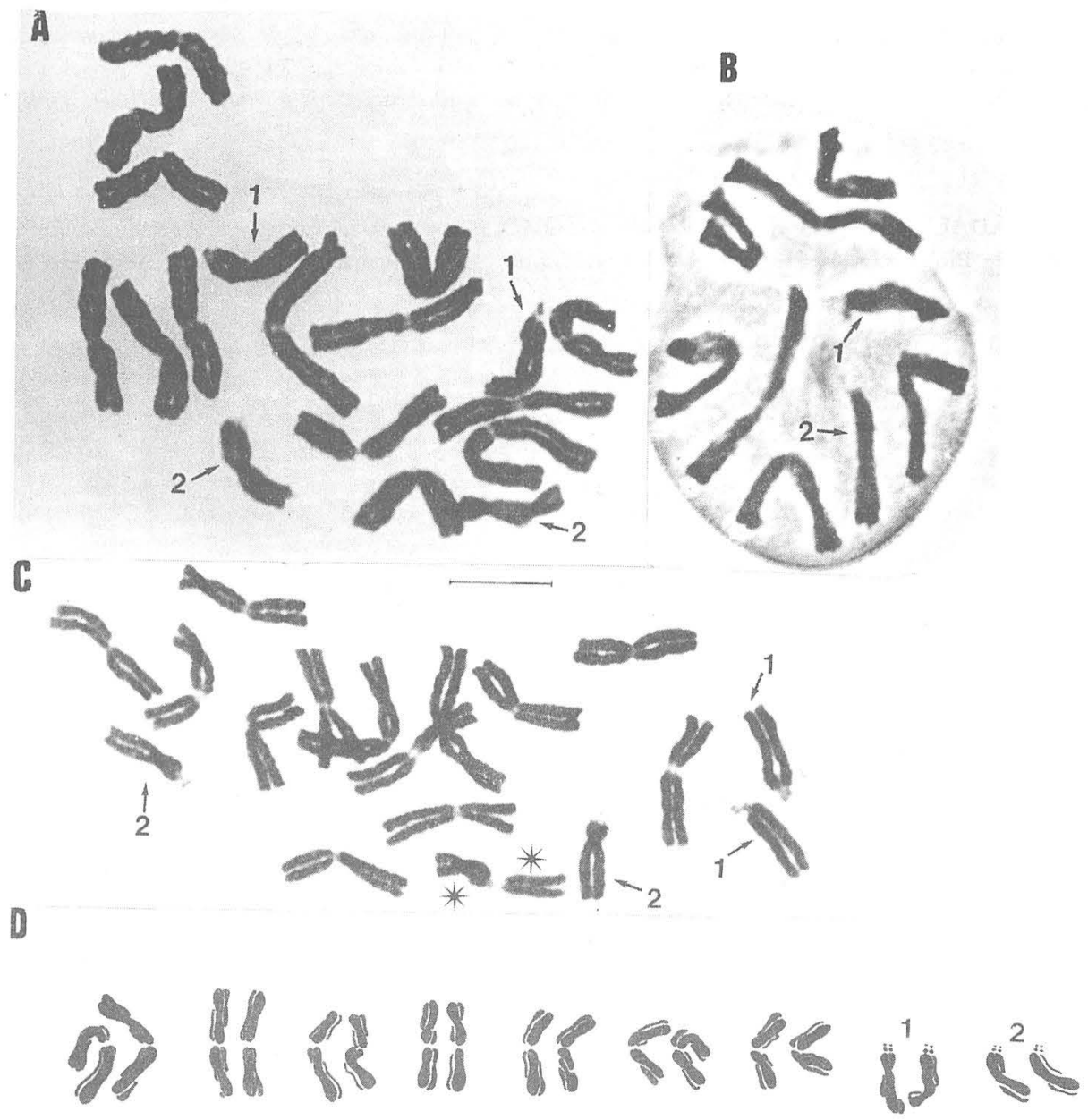

Fig. 1. Cromosomas y cariotipo de Nothoscordum bivalve. Los pares de los cromosomas telocéntricos 1 y 2 se señalan con flechas. A. Cromosomas somáticos de $N$. bivalve de una planta original, mostrando un complemento $2 n=18,14 \mathrm{M}+4 \mathrm{~T}$. B. Cromosomas de granos de polen en la primera mitosis, mostrando $n=9,7 \mathrm{M}+2 \mathrm{~T}$. C. Cromosomas somáticos de un clon de $N$. bivalve mostrando un complemento $2 n=19$ $(13 \mathrm{M}+6 \mathrm{~T})$. El par de cromosomas telocéntricos, generados por la fisión de un metacéntrico a nivel del centrómero se indican con asteriscos. D. Cariotipo de $N$. bivalve: $2 n=18,14 \mathrm{M}+4 \mathrm{~T}$. Las barras corresponden a $10 \mu$. 